\section{Sore Throat Culturing in Community Hospital Staff}

\section{To the Editor:}

The question of culturing "sore throats" of staff in community hospitals is an important one.' Obviously the protocol will impact on the well being of both staff and hospital.

E.P. Bradley Hospital is a children's psychiatric hospital with 300 employees and a census averaging 56 patients.

For many years employees have been cultured for the complaint of sore throat. The techniques are similar to that of Watanakunakorn with the addition of microbiological confirmation.

Between June 1981 and April 1985, 1,377 cultures of staff were taken and 234 were positive for beta hemolytic streptococcus group A representing an incidence rate of $17 \%$.

This is a much larger recovery rate than that found in the previously mentioned study.

The explanation of the difference may be the fact that our findings are from a child and adolescent hospital rather than a general hospital.

We feel that the conclusions recorded in the Watanakunakorn article are probably not generalizable.

\section{REFERENCES}

1. Watanakunakorn C: Should routine throat cultures be done in hospital personnel complaining of a sore throat? Infect Control 1985; 6:183-185.

John E. Farley, MD Director of Pediatrics Bradley Hospital East Providence, Rhode Island
Dr. Chatrchai Watanakunakorn responds to Dr. Farley's comments.

The higher recovery rate $(17 \%)$ of group A beta-hemolytic streptococcus from throat cultures of employees reported by Dr. Farley is most likely due to the fact that his hospital is a children's hospital. Our hospital is a general hospital with a very small pediatric patient population. Our finding $(6.2 \%)$ may not be applicable to employees of children's hospitals. Findings from other children's hospitals may be of interest.

Chatrchai Watanakunakorn, MD St. Elizabeth Hospital Medical Center Youngstown, Ohio

\section{To the Editor:}

In regard to the article "Should routine throat cultures be done in hospital personnel complaining of a sore throat?" by Dr. Chatrchai Watanakunakorn, ${ }^{1}$ pertaining to his statement that there is a lack of CDC guidelines for sore throat culturing of employees; on page 18 in the glossary of the Employee Health Guidelines, \#6 Personnel Restrictions Because of Illness for Special Conditions, \#6f Streptococcal Disease states:

If group A streptococcal disease is suspected, appropriate cultures should be taken, and the health worker should be excluded from work until she or he has received adequate therapy for 24 hours or until streptococcal infection has been ruled out. Category I. ${ }^{2}$

I also did a study prior to Dr. Watanakunakorn over a one year period because of the previously mentioned CDC recommendations. I included a cost analysis and compared it with nosocomial infections. Based on my findings, which were presented as an oral session at APIC's Cincinnati meeting this year, I made conclusions similar to Dr. Watanakunakorn's. My understanding is that this recommendation is being changed.

\section{REFERENCES}

1. Watanakunakorn C: Should routine throat cultures be done in hospital personnel complaining of a sore throat? Infect Control 1985; 6:183-185.

2. C.DK: Guidelines: Employee Heahh Guidelines, Centers for Disease Contml. Atlanta, Ceorgia. 1983.

Ted Cox, RN, MSPH, CIC Harry S. Truman Memorial V.A. Hospital Columbia, Missouri

\section{Amniotic Membranes for Burn Wounds}

\section{To the Editor:}

Regarding the request for references on the topic of use of amniotic membranes for burn wound surfaces (Letter to the Editor, August 1985), ${ }^{1}$ Dr. Baddour and others may find useful a short article on the subject with pictures of the handling and preparation of harvested membrane, in the April 1985 issue of the American Journal of Nursing. The article can be found in the Wound Care Forum section, written by P. Bower, RN, Vascular Nurse Clinician, Milwaukee County Medical Complex.

\section{REFERENCE}

1. Baddour LM: Infection control measures for the use of amniotic membranes. Infect Control 1985; $6: 300$.

Barbara Wyand Walker, RN, BS Infection Control Surveillance Nurse Humana Hospital Greenbrier Valley Ronceverte, West Virginia 